\title{
Initial Study on the State of the Art Solutions for the Simulation of the Thermoregulatory Sweating Conditions
}

\author{
Ioan Turcin ${ }^{1,3 *}$, Selver Softic ${ }^{2}$, Udo Traussnigg ${ }^{1}$, and Adrian Trif ${ }^{3}$ \\ ${ }^{1}$ CAMPUS 02 UAS/Degree Programmes in Automation Technology, 8010 Graz, Austria \\ ${ }^{2}$ CAMPUS 02 UAS/Degree Programmes in IT and Business Informatics, $8010 \mathrm{Graz}$, Austria \\ ${ }^{3}$ Technical University of Cluj-Napoca, Faculty of Machines Building, 400114 Cluj-Napoca, Romania
}

\begin{abstract}
The paper presents an initial study and basic research of the state of the art systems and solutions for the simulation of the thermoregulatory sweating conditions. The simulation of the thermoregulatory sweating conditions has been treated by many researchers, and has become over the years a major area of interest for many industries (e.g. textile, protective garments, aeronautics, automotive industries and sports). The objective of this paper is to reflect and analyse the existing literature, for this purpose. Three existing systems and two thermoregulatory models will be characterized and critically discussed. The findings support the methodology for future applied scientific research ( $\mathrm{PhD}$ thesis), which will use existing scientific knowledge to develop a practical application using suitable technology.
\end{abstract}

\section{Introduction}

Human body possess a complex thermoregulatory system. Under normal conditions the body keeps, the vital organs such as heart, lung and brain within a narrow temperature range around $37^{\circ} \mathrm{C}$. The body produces heat usually through metabolic processes (e.g. muscular activity). During such processes depends on climate circumstances and clothing worn, the body excesses the heat via evaporation, radiation, conduction and respiration [1].

According to the best knowledge of the authors, there is no known, price accessible, measuring equipment for the simulation of the thermoregulatory sweating available on the market for industrial applications, although many manikins and mathematical models have been developed in the last years.

To reproduce a physical, physiological situation or process conveniently, the developed mathematical model has to fit the studied situation. The existing systems are very sophisticated and specific, requiring high levels of training and qualification, which makes them inaccessible for small and medium enterprises (SMEs) with small R\&D departments and limited resources. Developing adequate, high qualitative and low-cost equipment,

${ }^{*}$ Corresponding author: ioan.turcin@campus02.at 
which can be adjusted to the demand of SMEs, would enable and motivate these companies to use these simulation systems.

The idea behind the future research ( $\mathrm{PhD}$ thesis) is to investigate and develop a prototype, using materials or material combinations that are still to be defined, and will lead to accurate thermal conductivity and humidity distribution simulations. Additionally, various advanced manufacturing methods will be investigated, which should allow the integration of sensing and measuring equipment, as well as fluid flow channels, in the tobe-produced prototype at minimal costs (Figure 1). A capable control algorithm based on suitable mathematical model making it easy to implement in future generation of thermal manikins, should accompany the developed element.

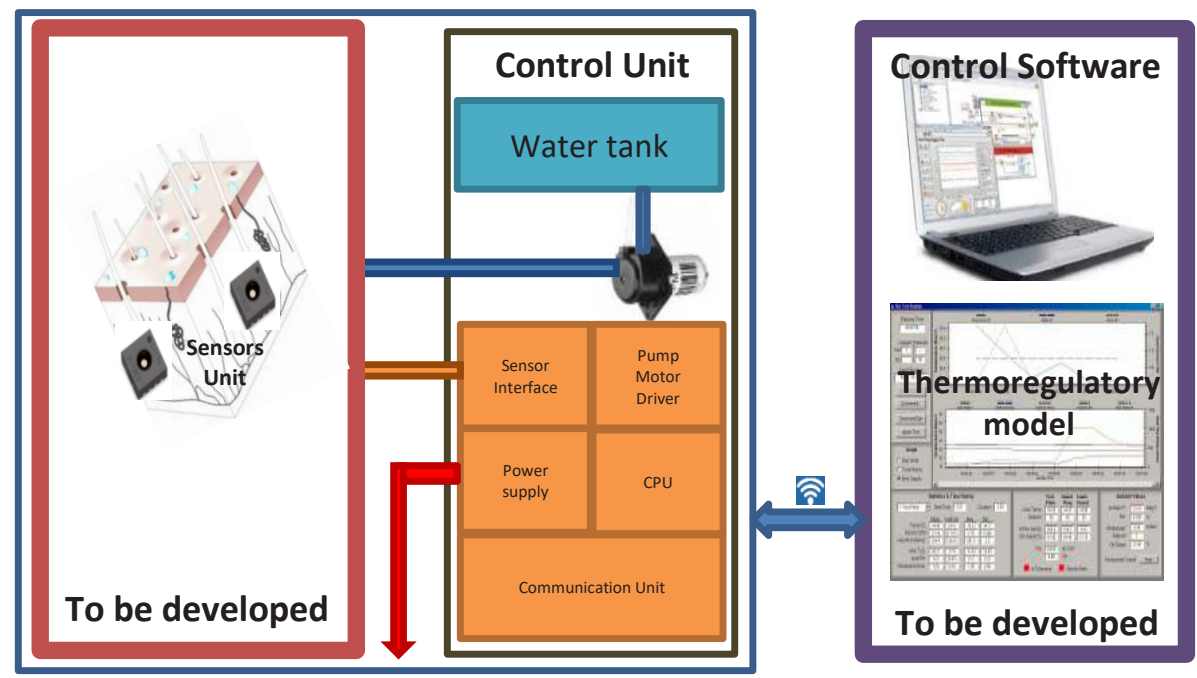

Fig. 1. The block diagramm of the prototype to be developed. The system should consist of three major elements: outer skin prepared by a combination of smart materials (e.g. two of them, sensors included), control unit (including heating, pumping system and needed interfaces) and a modern algorithm for the mathematical model of thermoregulation sweating conditions.

\section{Methodology and materials}

The intention behind this literature study is to reflect and analyse the developed techniques and state of the art used over the years to simulate the thermoregulatory sweating conditions. There is a large number of published studies about this topic. The focus of the search was on the scientific reflection of the intensely sought-after and found literature, where the information has been collected in a targeted manner. To cover many information and previous works, a variety of online catalogues, databases and open source libraries have been searched (e.g. Google Scholar, Directory of Open Access Journals, Science Direct, Management E-journals (Emerald), Elsevier, The Austrian Library Network and Service). The authors used following keywords for this operation: simulation, thermoregulatory, systems, sweating conditions, manikins and models of thermoregulation. After an intensive review, the materials with following reference number [1], [2], [3], [4], [5], [6] and [7], have been selected for further discussion. This choice criterion in particular was the holistic nature of approaches presented in found works. Only the papers covering all the fields of interest expressed by the searched key words mentioned above have been selected. At the end of selection and evaluation process, only complete and approved systems for simulation 
of thermoregulatory sweating systems have been considered for further observation and discussion.

\section{Discussion}

For many years, the developed technique of thermal hot plates or manikins have been used in successful technical applications. There are sweating thermal manikins, where the textile layer, are placed over the thermal manikin and are used for the simulation of the sweating. However, the measurements are too expensive and are not available for example for the SME's of the industry sector (e.g. sweating torso cost around 100.000,00 €). First used thermal manikin was early 1940s, which was a simple one segment copper made manikin introduced by the American army [3].

For a variety of applications in the engineering field, manikins (there are over 100 manikins in the world, which use thermoregulatory systems) are used for the evaluation of clothing thermal, evaporative resistance and similar applications. Advanced technologies have helped in designing of manikins to produce more realistic results than before relating to the human thermal interaction with the environment. In addition, advanced software and computing tools have enabled the combination of the modelling results with the obtained data from manikin tests.

\subsection{The manikins}

The selection of the manikins SAM (Switzerland), ADAM (USA), X-THERMOMAN (Slovenia) and Wissler (1985) and Fiala (2012) models, is based on the degree of brand awareness, complexity of the system, design of the sweating system used, analysis of the results, different countries of manufacture, etc. The selected manikins and models are introduced in the following paragraphs.

The SAM (Fig. 2) is a thermal manikin capable of simulating heavy work conditions, and human movements such as walking and climbing. The purpose was to simulate the human body in terms of heat production, sweat production and movement as closely as possible. The simulation system around SAM manikin allows observation of even small differences in clothing and possesses higher repeatability than those from human tests.
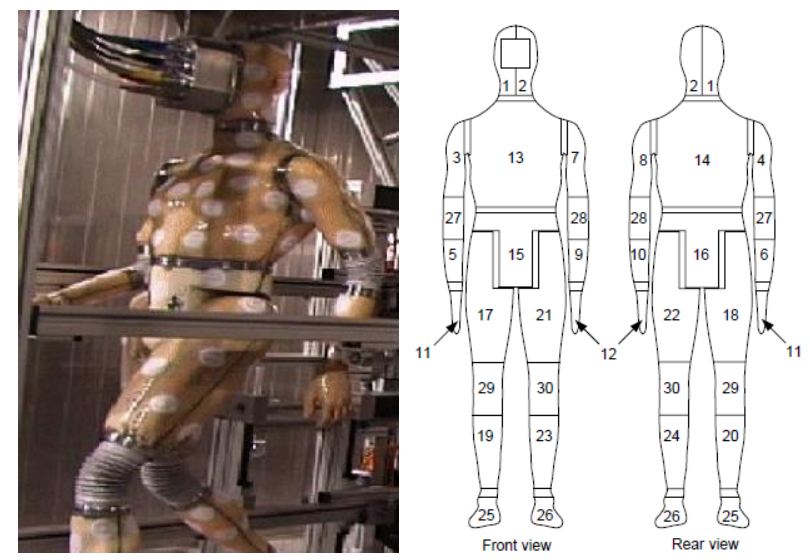

Fig. 2. Manikin SAM with his 30 separately heated sectors [1]. 
The complete system of SAM consists of 30 separate uniformly heated sectors. Each sector can be heated at a constant average surface temperature (to perform standard ISO TC $38 \mathrm{WG} 17$ ) or with a constant power (up to $1.2 \mathrm{~kW}$ ). SAM manikin simulation system allows performing measurements of thermal insulation or supplied with different heating powers to simulate various activities. Sweating system is made of 125 sweat outlets distributed over its surface. It also allows sweat rate regulation from $20 \mathrm{ml} / \mathrm{h}$ up to at least 4 litres per hour. In this way, it is possible to simulate various activities and conditions [1].

ADAM (Advanced Automotive Manikin) evaluates transient, non-uniform thermal environments in automobiles, where a sophisticated surface sensor interacts with his environment and is very good linked with a Human Thermal Physiological Model and a Human Thermal Comfort Model (Fig. 3). The manikin does not only respond to thermal inputs such as radiation and convection it also affects the environmental flow field and temperature field [2]. It is a three-dimensional transient finite element model, contains a detailed simulation of human internal thermal physiological systems and thermoregulatory responses.

ADAM consists of on-board power, water, communication, physiological control designed to respond to transient, non-uniform thermal environments like a human (sweating, breathing) and wears clothes, it is $175 \mathrm{~cm}$ tall, has $61 \mathrm{~kg}$, and contains 120 elements $\left(\sim 120 \mathrm{~cm}^{2}\right)$ and 120 zones. It provides a simulated body positioned in a complex thermal environment to measure the transient thermal response with extremely high spatial resolution.

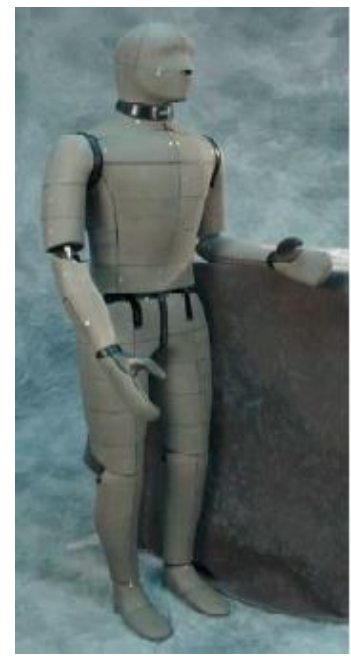

Fig. 3. Thermal manikin ADAM [2].

The finite element model integrated with ADAM provides the manikin with a control algorithm that represents human thermal response. The manikin can be considered as surface sensor that measures the rate of heat loss at each surface zone. The main objective of the ADAM manikin and its model system is to predict the effect of thermal environments on human thermal physiology [2].

X-TERMOMAN, presented manikin in the Figure 4, was the first manikin made at the Faculty of Mechanical Engineering in Maribor in Slovenia. 


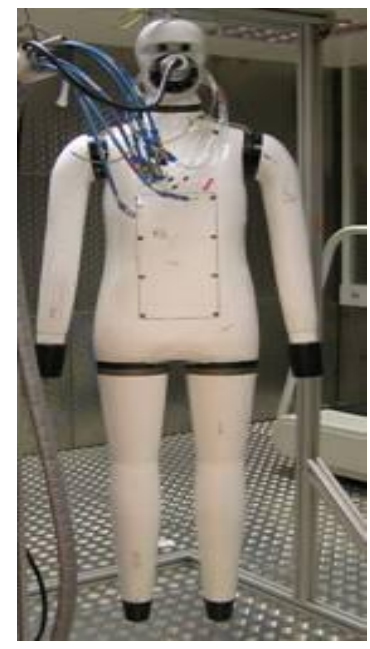

Fig. 4. X-termoman [3].

In order to approximate the desired copy of a human body as close as possible, the required heating power was set to $1000 \mathrm{~W}$, where the heaters are electrical (working on resistance principle) and the number of them is optimized in order to get the best possible heat distribution. The X-TERMOMAN was made of aluminium (AlSi9Cu3) which is a cost-efficient replacement for silver that was also protective garment considered as option. The choice fell on aluminium because of the need for thicker walls in order to ensure the self-carrying of the manikin's body [3].

There are significant differences between SAM, ADAM and X-TERMOMAN with respect to the application area, concept, topicality, materials, models of thermoregulation systems and technology used. SAM is capable of simulating movements and produce results, which have a much higher repeatability than those from human tests. On the other hand, ADAM has high spatial resolution, a reasonable response time, and realistic and uniform sweating rates. It is a new tool, which will be useful in predicting the thermoregulatory response of a person in a protective garment, which can also evaluate the effectiveness of personal cooling systems used with these types of suits [2]. Concerning XTERMOMAN (mostly used for protective garment), it is made of solid blocks of aluminium, selected based on a study of the thermal, mechanical and chemical properties of the material. It includes a self-developed suitable user interface designed to ensure a good overview of all crucial data and to enable simple and controlled testing according to ISO 15831 (2004) [3].

It is to mention, that only ADAM use the new wireless technology for communication between manikin and thermoregulatory model.

Thermal manikins will never really imitate the human and their results are approximate or indicative and can be used for relative tests. Suitable models may overcome limitations of design such as missing thermal capacity, blood flow control and thus a missing core temperature, but all of them reducing the need for expensive human tests. Tests under extreme conditions can be dangerous and they are strictly regulated by law. Using thermal manikins, enables performing the desired tests, without the possible risk of life. Considering the specific requirements and the aim of reducing the overall costs, the techniques are initially expensive to set up, therefore they are relatively exclusive. According to the current findings coming out of the study conducted, there are no standards yet for the materials used, design of the thermoregulatory sweating systems used and the analysis of the results. 


\subsection{Models of thermoregulation}

Some of the existing models of thermoregulation can be divided as follows:

Empirical models: Givoni and Goldman (1972-1973), Pandolf (1986), Malchaire (2000).

Rational models: Wissler (1961-1985), Stolwijk and Hardy 25-node model (1971), Nishi and Gagge 2-node model (1972), Haslam and Parsons, Kraning \& Gonzalez (1977), Fiala (1999-2012).

Wissler and Fiala are researchers who realized and described the existence of temperature gradients in the body and have contributed a lot to further development and research of thermoregulation models.

Wissler human thermoregulation model can be used within the areas of physiology, study of thermoregulation, simulation of the human, studying simulated thermoregulatory response and comparing them with real data of human. The continuously development over the past 50 years, was applied to simulate transient thermoregulatory behaviour for numerous environmental heat exchange conditions, types and levels of physical activity.

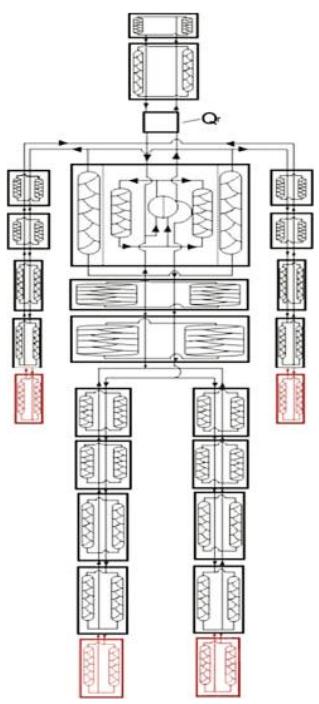

Fig. 5. Graphical representation of the classic Wissler model [4].

Recently, the Wissler human thermoregulation model has been finally translated into $\mathrm{C}$ code. The project possesses extensive internal documentation and the model in $\mathrm{C}$ is wrapped in Python, to provide a graphical user interface for convenient input and output operations [4].

Fiala considered 1999, that the reasons why regulatory processes did not became widespread, include lack of confidence in their predictive abilities, limited range of applicability, and poor modelling of the heat exchange with the environment. He intended to contribute to research efforts to formulate a more precise, flexible and universal model of the human thermoregulatory system [5]. Over the years, Fiala developed a new version called UTCI model (universal thermal climate index; active system), which is a new version of simplified DTS (Dynamic Thermal Sensation; passive system) models and can be seen in Figure 6. 


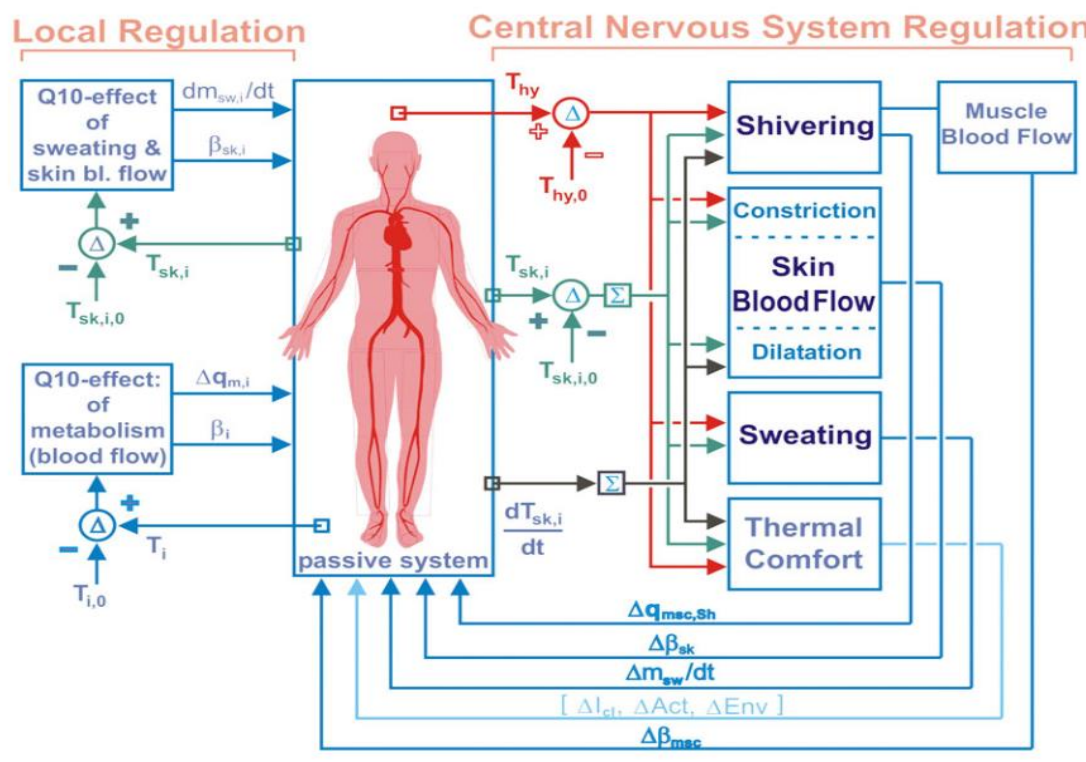

Fig. 6. Fiala's DTS and UTCI block diagram of active system [5].

The entire developed models shares characteristic, they have in common the modelling of the heat exchange within the body and the body with the environment.

Very important difference between the discussed models is in the modelling of the active part that determines physiological responses of the body. A very important distinguishing factor is that the models used different empirical relationships that correlate to diverse physiological responses (sweat rate factors, blood flow rates etc.) [7].

\section{Conclusion}

All discussed systems are very sophisticated, costly and do not offer the opportunity to focus only on the experiments on thermoregulatory sweating.

The large differences appear to be in apparatus (manikin and climatic chamber), experimental procedures, and methods of calculation used. In applying the different approaches for improving the thermal comfort, a holistic view should be considered and be creative in maximizing the functionality without compromising other important factors such aesthetics, cost, etc.

In order to different requirements and the aim of reducing the overall costs, many of the later manikin designs used plastics or even composite materials instead of metal. The metal manikins are still being manufactured for specific uses such as thermal comfort and heat loss analysis.

The findings of this study have a number of important implications for future practice because it provides a good overview of well-known systems and models and emphasize the missing gap for development of an affordable, general purpose, easily accessible and easy to use system for industrial applications.

The system aimed to be develop by the ongoing $\mathrm{PhD}$ thesis will focus on thermoregulatory sweating conditions and employ control algorithms based on developed mathematical models, as well as additive fabrication techniques that will allow it to be flexible, modular and customizable. 
This research was supported by the OpTi-DeP Project (no. BG101/2016) financed from the UEFISCDI by the Romanian Government and by the HORIZON 2020 AMaTUC project (GA 691787) financed from EU.

\section{References}

1. M.G.M. Richards, N.G. Mattle, Def Tech Inf Cent, Comp Pt Not ADP012411, 4-2, 44, 4-5 (2002)

2. R.B. Farrington, J.P. Rugh, D. Bharathan, R. Burke, SAE Int, 2004-01-2345, 1, 2, 5, (2004)

3. I. Pahole, B. Valentan, D. Z. Pavlinić, M. Ficko, J. Balič, T 22(6), 1623-1626 (2015)

4. K. R. Diller, D. W. Hensley, A. E. Mark, J. R. Abella, G. M. Netscher, E. H. Wissler, J of Biomech Eng 135, 021005-4, 021005-8 (2013)

5. J. C. Guedes, Mathematical Modelling of Human Thermoregulation in Hot Environments, PhD Thesis, (2016)

6. D. Fiala, K. J. Lomas, and M. Stohrer, Int J Biometeorol 45, 144 (2001)

7. K. Katic, R. Li, W. Zeiler, Elsevier Build \& Env 106, 296 (2016) 